\title{
Fatores associados à atividade física de lazer entre idosos do município de São Paulo
}

\section{Factors associated with leisure-time physical activity among elderly in São Paulo \\ Factores asociados con la actividad física en el ocio entre personas mayores del municipio de São Paulo}

\author{
Bruno Holanda Ferreira (iD \\ Universidade de São Paulo - São Paulo (SP) - Brasil \\ Bruna Gabriela Marques (iD \\ Universidade São Judas Tadeu - São Paulo (SP) - Brasil \\ Olinda do Carmo Luiz (iD \\ Universidade de São Paulo - São Paulo (SP) - Brasil
}

\section{RESUMO}

Objetivo: Analisar a prevalência de atividade física de lazer em nível suficiente (AFLS) entre idosos residentes no município de São Paulo e sua associação com características sociodemográficas, comportamentos de saúde e morbidade. Métodos: Trata-se de um estudo transversal com uma amostra de 693 idosos ( $\geq 60$ anos de idade) residentes no município de São Paulo, Brasil, cujos dados sociodemográficos, comportamento em saúde e morbidade foram extraídos a partir do sistema Vigilância de Fatores de Risco e Proteção para Doenças Crônicas por Inquérito Telefônico, referente ao ano de 2017. Realizou-se estatística descritiva, testes de associação pelo qui-quadrado, regressão logística simples bruta e ajustada, por idade, sexo e escolaridade, assim como regressão múltipla. Resultados: A prevalência de AFLS entre os idosos entrevistados foi de $22,5 \%$ ( $n=156)$. Entre os idosos com escolaridade $>12$ anos houve maior chance de ser ativo (OR=2,47; IC95\% 1,58-3,85) em relação àqueles com 0-8 anos de estudo, bem como entre os que apresentaram estado de saúde "bom" (OR=2,96; IC95\%1,12-7,76) e "muito bom" $(\mathrm{OR}=5,14 ;$ IC95\%1,86-14,23) em relação àqueles que relataram possuir saúde "ruim/muito ruim/não sabe". Conclusão: No contexto geral, pode-se inferir que um a cada quatro idosos moradores do município de São Paulo praticam atividade física suficiente no lazer. Entre os idosos, a prevalência foi maior entre os mais jovens, do sexo masculino, com companheiro, branco, maior escolaridade, com ocupação, que relataram estado de saúde "muito bom", referiram não possuir doenças crônicas, consumir bebida alcoólica e ser não fumante.

Descritores: Saúde do Idoso; Envelhecimento; Promoção da Saúde; Estudos Transversais.

\section{ABSTRACT}

Objective: Analyze the prevalence of Leisure-time Physical Activity in the Sufficient Leisure Domain (PASL) among elderly people living in the city of São Paulo and its association with sociodemographic characteristics, health behaviors, and morbidity. Methods: This is a cross-sectional study with a sample of 693 elderly people ( $\geq 60$ years old), living in the city of São Paulo, Brazil, whose sociodemographic data, health behavior and morbidity were extracted from the system Surveillance of Risk and Protective Factors for Chronic Diseases through Telephone Survey, referring to the year 2017. Descriptive statistics, association tests by chi-square, simple crude and adjusted logistic regression, by age, gender, and education, as well as multiple regressions were performed. Results: Prevalence of PASL among the elderly interviewed was $22.5 \%(n=156)$. Among the elderly with education $>12$ years, there was a greater chance of being active $(O R=2.47 ; 95 \% \mathrm{Cl} 1.58-3.85)$ in relation to those with $0-8$ years of study, as well as among those who presented a "good" health condition (OR=2.96; 95\% Cl 1.12-7.76) and "very good" (OR=5.14; $95 \% \mathrm{Cl}$ 1.86-14.23) in relation to those who reported having "Bad/very bad health/don't know". Conclusion: In the general context, it can be inferred that one in four elderly people living in the city of São Paulo practices enough physical activity during leisure. Among the elderly, the prevalence was higher among the younger ones, male, with a partner, white, higher education, with an occupation, which reported "very good" health condition, reported not having chronic diseases, consuming alcohol, and not being smoker.

Descriptors: Health of the Elderly; Aging; Health Promotion; Cross-Sectional Studies. 


\section{RESUMEN}

Objetivo: Analizar la prevalencia de actividad física en el ocio en nivel suficiente (AFOS) entre mayores del municipio de São Paulo y su asociación con las características sociodemográficas, las conductas de salud y la morbilidad. Métodos: Se trata de un estudio transversal con la muestra de 693 mayores ( $\geq 60$ años de edad) del municipio de São Paulo, Brasil, cuyos datos sociodemográficos, las conductas de salud y la morbilidad fueron sacados del sistema de Vigilancia de Factores de Riesgo y Protección para Enfermedades Crónicas por Encuesta Telefónica referente al año 2017. Se realizó la estadística descriptiva, las pruebas de asociación por el chi-cuadrado, la regresión logística simple bruta y ajustada para la edad, el sexo y la escolaridad así como la regresión múltiple. Resultados: La prevalencia de la AFOS entre los mayores entrevistados ha sido del $22,5 \%$ ( $n=156$ ). Entre los mayores con más de 12 años de escolaridad hubo más oportunidad de ser activo (OR=2,47; IC95\% 1,58-3,85) que aquellos con hasta 8 años de estudio así como entre los que presentaron el estado de salud "bueno" $(O R=2,96 ;$ IC95\%1,12-7,76) y "muy bueno" (OR=5,14; IC95\%1,86-14,23) que aquellos que relataron tener "mala/muy mala" salud o "no sabe". Conclusión: En el contexto general se puede decir que uno de cada cuatro mayores del municipio de São Paulo practica actividad física suficiente en el ocio. Entre los mayores, la prevalencia ha sido mayor entre los más jóvenes, del sexo masculino, con pareja, de color blanco, con más escolaridad y con ocupación que han relatado el estado de salud "muy bueno", no tener enfermedades crónicas, no tomar bebida alcohólica y no ser fumador.

Descriptores: Salud del Anciano; Envejecimiento; Promoción de la Salud; Estudios Transversales.

\section{INTRODUÇÃO}

Nas últimas décadas, tem-se observado o envelhecimento populacional no Brasil e no mundo. Em 2017, estimou-se que existiam 962 milhões $(13 \%)$ de pessoas com idade igual ou superior a 60 anos ${ }^{(1)}$. O processo de envelhecimento teve inicio há mais de um século nas nações desenvolvidas e avança no cenário dos países em desenvolvimento, de modo que, na Europa, $25 \%$ da população é constituída por idosos, já na América latina, em países como Venezuela e Peru, a proporção é de 10\%; no Chile, $16 \%$; no Brasil, $13 \%$ e, no Uruguai, $20 \%{ }^{(1)}$. Uma das justificativas para esse crescimento demográfico é, principalmente, a redução da mortalidade e da natalidade, com consequente aumento da expectativa de vida ${ }^{(1)}$.

Dentro do cenário acima descrito, é notória a preocupação e a necessidade de ampliar e aprimorar a atenção à saúde da população idosa, levando em consideração as alterações multifacetadas e complexas que ocorrem com a idade, que acarretam a diminuição e/ou alteração da composição muscular, da massa óssea ${ }^{(2)}$ e da capacidade cardiorrespiratória ${ }^{(3)}$, comprometendo o desempenho de diversas tarefas. Além disso, os idosos precisam de tratamentos farmacológicos por conviver com diversas patologias crônicas e incapacidades física, cognitiva e mental, fragilidades que levam ao comprometimento da qualidade de vida ${ }^{(4)}$.

A expansão das doenças crônicas na maior parte dos países, relacionada ao estilo de vida não saudável da vida moderna (caracterizado pelo consumo de tabaco e álcool, inatividade física, alimentação não saudável e obesidade), orientou a formulação de estratégias preventivas em prol do enfrentamento das doenças crônicas não transmissíveis $(\text { DCNT })^{(5,6)}$. Mesmo assim, em 2017, a morte entre indivíduos de todas as idades por DCNT no mundo representou $73 \%$, isto é, mais da metade de todas as mortes ( 28,8 milhões) são atribuíveis a quatro características de risco: pressão alta, tabagismo, glicemia alta e alto índice de massa corporal( ${ }^{(6)}$.

Com o objetivo de minimizar a incapacidade física prematura, prevenir ou tratar as DCNT no processo de envelhecimento, destaca-se o efeito protetor da atividade física, que, quando praticada de maneira regular, pode melhorar as funções cardiovasculares ${ }^{(7,8)}$, a função física ${ }^{(9)}$, diminuir a dor ${ }^{(10)}$ e os sintomas de ansiedade e depressão(11,12), contribuindo no desenvolvimento e/ou manutenção de habilidades funcionais para o envelhecimento saudável ${ }^{(13)}$.

Apesar dos benefícios apontados em relação à prática regular de atividade física e do envelhecimento fisiológico depender do estilo de vida que a pessoa assume desde a infância, estima-se que $27,5 \%$ dos indivíduos adultos no mundo não atingem a recomendação de realizar, no mínimo, 150 minutos por semana de atividade física de intensidade moderada ou equivalente ( $\geq 75$ minutos por semana com nível intenso ou vigoroso, ou mesmo, a combinação das duas intensidades) $)^{(14)}$, contribuindo com 1.26 milhões de mortes no ano de $2017^{(15)}$.

No Brasil, observa-se um aumento anual de indivíduos ativos no lazer, subindo de 44\%, em 2006, para 53,6\%, em 2016 (média de 0,97pp/ano)(16). Nessa ocasião, a atividade física entre os idosos passou de 31,8\%, em 2006, para 40,5\%, em 2016 (média de 0,56 pp/ano), mas acarretando o menor crescimento entre todas as faixas etárias ${ }^{(16)}$.

Apesar disso, poucos são os estudos de base populacional que investigaram a prevalência de AFLS entre a população idosa brasileira ${ }^{(16-18)}$, mais especificamente em regiões de São Paulo(19-22), evidenciando a necessidade de 
exploração nacional. Com isso, o presente estudo objetiva analisar a prevalência de atividade física no lazer suficiente entre os idosos residentes no município de São Paulo e sua associação com características sociodemográficas, comportamento de saúde e morbidade.

\section{MÉTODOS}

Trata-se de um estudo transversal, realizado entre fevereiro e julho de 2019, cujos dados foram obtidos a partir do sistema de Vigilância de Fatores de Risco e Proteção para Doenças Crônicas por Inquérito Telefônico (Vigitel), coletadas entre janeiro e dezembro de 2017. A entrevista do Vigitel possui um sistema no qual as perguntas são lidas na tela do computador e suas respectivas respostas registradas diretamente em meio eletrônico, permitindo pulos automáticos de questões não válidas decorrentes das respostas anteriores e minimizando erros de digitação(23).

O procedimento amostral empregado pelo Vigitel visa obter amostra probabilística da população com 18 anos ou mais de idade; cadastradas em, pelo menos, uma linha de telefone fixo residencial no território brasileiro; obtidas em duas etapas: a) sorteio sistemático de 5.000 linhas telefônicas em cada cidade, seguidas por ressorteio e organização de 25 subamostras de 200 linhas, b) sorteio de um morador adulto do domicilio para responder à entrevista ${ }^{(23,24)}$. Essas estratificações buscam igualar as distribuições sociodemográficas da amostra a partir de projeções da distribuição nos Censos Demográficos de 2000 e 2010 e sua variação média no período intercensitário. O sistema apresenta métodos próprios, que podem ser encontrados em publicação do Vigitel(24).

Em 2017, o Vigitel entrevistou, no município de São Paulo, 2.052 adultos, sendo 766 homens e 1.286 mulheres. Desse total, foram elegíveis para este estudo 693 indivíduos, com idade $\geq 60$ anos (223 homens e 470 mulheres). O Sistema Estadual de Análise de Dados (Seade) ${ }^{(25)}$ estimou, no ano considerado, que 14,3\% (1.676.101) era composto por pessoas com idade $\geq 60$ anos no município de São Paulo.

A variável de desfecho obtida a partir da atividade física de lazer (AFL) possui a seguinte classificação categórica: atividade física de lazer suficiente (AFLS) - indivíduo que praticou $\geq 150$ ou $\geq 75$ minutos semanais de atividade física de intensidade moderada ou vigorosa, e atividade física de lazer insuficiente (AFLI) - o indivíduo que não atingiu o tempo em minutos, de acordo com os critérios adotados pelo Vigitel do ano de $2010^{(24)}$. Estima-se essa variável por meio de cinco questões: "Nos últimos três meses, o(a) sr(a) praticou algum tipo de exercício físico ou esporte?", "Qual o tipo principal de exercício físico ou esporte que o (a) $\operatorname{sr}(a)$ praticou?", "O(a) sr(a) pratica o exercício pelo menos uma vez por semana?", "Quantos dias por semana o(a) sr(a) costuma praticar exercício físico ou esporte?" e "No dia em que o(a) sr(a) pratica exercício ou esporte, quanto tempo dura esta atividade?".

Em relação às variáveis de exposição, optou-se por investigar as características demográficas e socioeconômicas relacionadas à faixa etária (60-69, 70-79 e $\geq 80$ anos), sexo (masculino e feminino), relação conjugal (com companheiro, sem companheiro e não respondeu/sabe), raça/cor (branca e não branca), escolaridade em anos (0-8, 9-11 e $\geq 12$ ), ocupação (sim - trabalha e não - não trabalha).

Para avaliação do estado de saúde, optou-se por selecionar as variáveis de autoavaliação do estado de saúde ("muito bom", "bom", "regular", "ruim/muito ruim/não sabe/não quis informar") e a exposição a fatores de risco modificáveis: consumo de álcool e tabagismo (sim ou não) e diagnóstico de DCNT: hipertensão e diabetes (sim ou não).

Para a análise estatística, realizou-se a distribuição da frequência absoluta e relativa de todas as variáveis e, em seguida, segundo AFLS, o teste qui-quadrado foi utilizado para analisar a associação entre as variáveis de exposição e AFLS. As variáveis que apresentaram nível de significância de $p<0,20$ no qui-quadrado foram inseridas na análise de regressão logística para estimar o odds ratio (OR) e os respectivos intervalos de $95 \%$ de confiança (IC95\%) e, em seguida, ajustando-os segundo idade, sexo e escolaridade para controlar a influência dessas variáveis nas estimativas. Selecionaram-se as variáveis que apresentaram valor de $p<0,20$ no modelo ajustado para a análise de regressão logística múltipla, sendo mantidas as variáveis com o nível de significância menor que $5 \%$. Todas as análises receberam o tratamento estatístico através do pacote Statistical Package for the Social Sciences (SPSS), versão 20.0.

Por se tratar de entrevistas por telefone, o consentimento livre e esclarecido foi substituído pelo consentimento verbal obtido por ocasião dos contatos telefônicos com os entrevistados, obtido pelo Ministério da Saúde no momento da entrevista. O projeto de implantação do Vigitel obteve aprovação do Comitê Nacional de Ética em Pesquisa para Seres Humanos do Ministério da Saúde (Parecer n. ${ }^{\circ} 355.590$, de 26/6/2013).

O banco de dados é público, não permite a identificação dos entrevistados e está disponível em: http://svs.aids. gov.br/bases_vigitel_viva/vigitel.php. 


\section{RESULTADOS}

A amostra final do estudo foi composta por 693 idosos, com média etária de 70,9 anos $( \pm 8,0)$, variando entre 60 e 95 anos. A Tabela I apresenta a descrição das variáveis analisadas, isto é, faixa etária, sexo, relação conjugal, raça/cor, escolaridade (anos) e ocupação. Os resultados indicam uma prevalência de indivíduos com faixa etária mais jovem (48,3\%; $n=335)$, do sexo feminino $(67,8 \%$; $n=470)$, sem companheiro $(56,4 \%$; $n=391)$ e branco $(65,2 \%$; $\mathrm{n}=452)$. Em relação à escolaridade e ocupação, $60,9 \%(n=422)$ dos entrevistados possuem entre $0-8$ anos de estudo e $69,3 \%(n=480)$ não têm ocupação.

No geral, 22,5\% ( $n=156)$ dos entrevistados declararam praticar AFLS (Tabela I). Observa-se que a população idosa de $60-69$ anos $(25,4 \% ; n=85)$ e homens $(22,9 \% ; n=51)$ possuem maior proporção de ativos no lazer, em relação à população mais idosa, com idade $\geq 80$ anos $(16,3 \% ; n=20)$, e mulheres $(22,3 \% ; n=105)$. Nas variáveis estado conjugal, raça/cor, escolaridade (anos) e ocupação, nota-se prevalência de AFLS nos participantes com companheiro (26\%; $n=83)$, branco (25\%; $n=113)$, escolaridade igual e/ou maior que 12 anos $(38,1 \% ; n=553)$ e com ocupação (26,3\%; n=56). A análise do qui-quadrado demonstrou haver associação de AFL com as variáveis faixa etária, relação conjugal, raça/cor, escolaridade (anos) e ocupação $(p<0,20)$.

A Tabela II apresenta prevalência de $43,7 \%(n=303)$ entre os idosos que relataram estado de saúde bom. Entre aqueles que referiram doenças crônicas, ter pressão elevada $(54 \% ; n=374)$ e ter diabetes $(78,5 \%$; $n=544)$ foram prevalentes no estudo. Os fatores de risco modificáveis relacionados aos hábitos apresentaram prevalência superior entre os idosos que declararam não consumir bebida alcoólica e não ser tabagista, com 76,2\% $(n=528)$ e $90 \%(n=624)$, respectivamente. Entre os pesquisados que relataram ser ativos no lazer, é possível notar prevalência entre a percepção do estado de saúde "muito bom" $(41,4 \% ; n=41)$, não ter pressão elevada $(27,3 \% ; n=87)$ nem diabetes $(24,4 \% ; n=133)$, não ter hábito de ingerir álcool $(29,7 \% ; n=49)$ e não ser tabagista $(23,2 \% ; n=145)$. Além disso, observa-se que todas as variáveis foram associadas à AFL, Tabela II.

Tabela I - Prevalência total entre os idosos (+ de 60 anos) praticantes de atividade física no lazer segundo características sociodemográficas. São Paulo, Vigitel-2017, n=693.

\begin{tabular}{|c|c|c|c|c|c|}
\hline \multirow{2}{*}{ Variáveis e categorias } & \multicolumn{2}{|c|}{ Total } & \multicolumn{2}{|c|}{ AFLS } & \multirow[b]{2}{*}{$p^{*}$} \\
\hline & $\mathbf{n}$ & $\%$ & $\mathbf{n}$ & $\%$ & \\
\hline Faixa etária & & & & & 0,110 \\
\hline $60-95$ & 663 & 100 & 156 & 22,5 & \\
\hline $60-69$ & 335 & 48,3 & 85 & 25,4 & \\
\hline $70-79$ & 235 & 33,9 & 51 & 21,7 & \\
\hline$\geq 80$ & 123 & 17,7 & 20 & 16,3 & \\
\hline Sexo & & & & & 0,876 \\
\hline Feminino & 470 & 67,8 & 105 & 22,3 & \\
\hline Masculino & 223 & 32,2 & 51 & 22,9 & \\
\hline Relação conjugal & & & & & 0,184 \\
\hline Sem companheiro & 391 & 56,4 & 70 & 19,3 & \\
\hline Com companheiro & 291 & 42 & 83 & 26 & \\
\hline Não respondeu & 11 & 1,6 & - & - & \\
\hline Raça/cor & & & & & 0,099 \\
\hline Branco & 452 & 65,2 & 113 & 25 & \\
\hline Não branco & 162 & 23,4 & 29 & 17,9 & \\
\hline Não respondeu & 79 & 11,4 & 14 & 17,7 & \\
\hline Escolaridade (anos) & & & & & $<0,001$ \\
\hline $0-8$ & 422 & 60,9 & 70 & 16,6 & \\
\hline $9-11$ & 132 & 19,0 & 33 & 25 & \\
\hline$\geq 12$ & 139 & 20,1 & 553 & 38,1 & \\
\hline Ocupação & & & & & 0,112 \\
\hline Não & 480 & 69,3 & 100 & 20,8 & \\
\hline Sim & 213 & 30,7 & 56 & 26,3 & \\
\hline
\end{tabular}

AFLS = atividade física de lazer suficiente; *Teste de independência (qui-quadrado) que se refere à associação da variável de exposição ao desfecho 
Tabela II - Prevalência total entre os idosos (+ de 60 anos) praticantes de atividade física no lazer segundo comportamentos de saúde e morbidades. São Paulo, Vigitel-2017, n=693.

\begin{tabular}{|c|c|c|c|c|c|}
\hline \multirow{2}{*}{ Variáveis e categorias } & \multicolumn{2}{|c|}{ Total } & \multicolumn{2}{|c|}{ AFLS } & \multirow[b]{2}{*}{$\mathbf{p}^{*}$} \\
\hline & $\mathbf{n}$ & $\%$ & $\mathbf{n}$ & $\%$ & \\
\hline Estado de saúde & & & & & $<0,001$ \\
\hline Ruim/muito ruim/não sabe & 50 & 7,2 & 5 & 10 & \\
\hline Regular & 241 & 34,8 & 30 & 12,4 & \\
\hline Bom & 303 & 43,7 & 80 & 26,4 & \\
\hline Muito bom & 99 & 14,3 & 41 & 41,4 & \\
\hline Pressão elevada & & & & & 0,005 \\
\hline Não & 319 & 46 & 87 & 27,3 & \\
\hline Sim & 374 & 54 & 69 & 18,4 & \\
\hline Diabetes & & & & & 0,019 \\
\hline Não & 544 & 78,5 & 133 & 24,4 & \\
\hline $\operatorname{Sim}$ & 149 & 21,5 & 23 & 15,4 & \\
\hline Consumo de álcool & & & & & 0,036 \\
\hline Não & 528 & 76,2 & 108 & 20,3 & \\
\hline Sim & 165 & 23,8 & 49 & 29,7 & \\
\hline Tabagista & & & & & 0,168 \\
\hline Não & 624 & 90 & 145 & 23,2 & \\
\hline Sim & 69 & 10 & 11 & 16 & \\
\hline
\end{tabular}

AFLS = atividade física de lazer suficiente; * Teste de independência (qui-quadrado) que se refere à associação da variável de exposição ao desfecho

O resultado da regressão logística binária bruta indica haver associação entre AFLS e as variáveis a seguir: faixa etária, estado conjugal, raça/cor, escolaridade (anos), ocupação, estado de saúde, pressão elevada, diabetes, consumo de álcool e tabagismo. No entanto, após o ajuste por sexo, escolaridade (anos) e faixa etária, permaneceram no modelo somente as variáveis escolaridade (anos), estado de saúde, pressão elevada e diabetes ( $<<0,20$ ), Tabela III.

A Tabela IV apresenta os resultados da análise multivariada. Verificou-se associação estatisticamente significativa de AFLS com escolaridade (anos) e estado de saúde $(p \leq 0,05)$. Utilizando-se como medida de associação a razão de chance (odds ratio, OR), observou-se entre idosos com escolaridade $\geq 12$ anos chance 2,47 vezes (IC95\% 1,583,85 ) maior de ser ativo no lazer quando comparados com aqueles que apresentaram escolaridade de 0-8 anos. Além disso, tendo como referência de comparação ter estado de saúde "ruim/muito ruim/não sabe", observou-se chance 2,96 vezes (IC95\% 1,12-7,76) maior entre os que relataram estado de saúde "bom" e de 5,14 vezes (IC95\% $1,86-14,23)$ maior entre os que relataram "muito bom".

\section{DISCUSSÃO}

O presente estudo apontou que possuir escolaridade $\geq 12$ anos e relatar estado de saúde "muito bom" e "bom" estão associados à prática de AFLS entre idosos residentes no município de São Paulo da amostra em questão, o que reforça a hipótese de causalidade. Os resultados encontrados por meio do décimo segundo ano de monitoramento do Vigitel, demonstram interessantes reflexões sobre as variáveis sociodemográficas, morbidades e hábitos, bem como o aprofundamento sobre elas, além de contribuir para o acúmulo de conhecimento no comportamento da população envelhecida.

Vale lembrar que, de uma maneira geral, tem-se observado um aumento nos níveis de atividade física de lazer no mundo ${ }^{(26)}$ e na população brasileira ${ }^{(16,27)}$. No entanto sabe-se que, com o processo de envelhecimento, o aumento nos níveis de atividade física de lazer é inferior quando comparado aos adultos ao longo dos anos ${ }^{(16)}$, chegando a ocorrer um decréscimo entre a população de idosos. No presente estudo, os resultados apontaram que menos de um quarto $(22,5 \%)$ da população da amostra é ativa no lazer.

Estudos na região de São Paulo envolvendo a população idosa apresentaram variação maior $\left(28,4 \%{ }^{(22)}\right.$ e $\left.25,3 \%{ }^{(17)}\right)$ e menores $\left(21,3 \%^{(19)}, 22,2 \%^{(21)}\right.$ e $\left.15,2 \%^{(20)}\right)$. Já em Florianópolis, Santa Catarina, a proporção de idosos ativos no lazer foi de $25,7 \%^{(28)}$; em Maceió, Alagoas, de $23,8 \%{ }^{(29)}$, e de $33 \%$ em 15 países europeus ${ }^{(30)}$. Logo, a prevalência observada na atual pesquisa foi semelhante à média nacional entre os anos de 2009 a $2016^{(13)}$.

Sabe-se que a prática de atividade física de lazer deve ser realizada por qualquer indivíduo( ${ }^{(31)} \mathrm{e}$, em especial, pela população idosa ${ }^{(7,8,31-33)}$. A literatura gerontológica enfatiza que o grupo etário dos 60 a 79 anos ${ }^{(16)}$ e do sexo masculino ${ }^{(17)}$ tem uma relação positiva para AFLS ${ }^{(16)}$. No presente estudo, observou-se que, após o ajuste estatístico, não foi possivel estabelecer a diferença entre os grupos etários e o sexo. 
Tabela III - Análise de regressão logística simples dos idosos (+ de 60 anos) praticantes de atividade física no lazer segundo variáveis sociodemográficas, comportamentos de saúde e morbidades. São Paulo, Vigitel-2017, n=693.

\begin{tabular}{|c|c|c|c|c|}
\hline Categorias e variáveis & OR bruto (IC95\%) & $\mathbf{p}$ & ${ }^{*}$ OR ajustado (IC95\%) & $\mathbf{p}$ \\
\hline Faixa etária & & 0,113 & & 0,426 \\
\hline 60-69 & 1,00 & & 1,00 & \\
\hline $70-79$ & $0,81(0,54-1,21)$ & & $0,89(0,59-1,34)$ & \\
\hline$\geq 80$ & $0,57(0,33-0,97)$ & & $0,69(0,40-1,20)$ & \\
\hline Sexo & & 0,876 & & 0,622 \\
\hline Feminino & 1,00 & & 1,00 & \\
\hline Masculino & $1,03(0,70-1,50)$ & & $0,90(0,61-1,34)$ & \\
\hline Estado conjugal & & 0,185 & & 0,513 \\
\hline Sem companheiro & 1,00 & & 1,00 & \\
\hline Com companheiro & $1,47(1,02-2,11)$ & & $1,31(0,87-1,98)$ & \\
\hline Raça/cor & & 0,101 & & 0,601 \\
\hline Branco & 1,00 & & 1,00 & \\
\hline Não branco & $0,65(0,41-1,03)$ & & $0,79(0,49-1,27)$ & \\
\hline Escolaridade (anos) & & $<0,001$ & & $<0,001$ \\
\hline $0-8$ anos & 1,00 & & 1,00 & \\
\hline $9-11$ anos & $1,67(1,04-2,68)$ & & $1,65(1,03-2,65)$ & \\
\hline$>12$ anos & $3,09(2,02-4,75)$ & & $2,99(1,93-4,64)$ & \\
\hline Ocupação & & 0,113 & & 0,994 \\
\hline Não & 1,00 & & 1,00 & \\
\hline $\operatorname{Sim}$ & $1,35(0,93-1,97)$ & & $1,00(0,65-1,53)$ & \\
\hline Estado de saúde & & $<0,001$ & & $<0,001$ \\
\hline Ruim/muito ruim/não sabe & 1,00 & & 1,00 & \\
\hline Regular & $1,28(0,47-3,48)$ & & $1,28(0,47-3,51)$ & \\
\hline Bom & $3,23(1,24-8,42)$ & & $2,94(1,11-7,77)$ & \\
\hline Muito bom & $6,37(2,32-17,41)$ & & $5,13(1,85-14,25)$ & \\
\hline Pressão elevada & & $<0,006$ & & 0,031 \\
\hline Não & 1,00 & & 1,00 & \\
\hline Sim & $0,60(0,42-0,86)$ & & $0,66(0,46-0,96)$ & \\
\hline Diabetes & & 0,021 & & 0,077 \\
\hline Não & 1,00 & & 1,00 & \\
\hline $\operatorname{Sim}$ & $0,56(0,34-0,91)$ & & $0,63(0,38-1,05)$ & \\
\hline Consumo de álcool & & 0,043 & & 0,573 \\
\hline Não & 1,00 & & 1,00 & \\
\hline Sim & $1,65(1,11-2,46)$ & & $1,28(0,83-1,96)$ & \\
\hline Tabagista & & 0,172 & & 0,604 \\
\hline Não & 1,00 & & 1,00 & \\
\hline Sim & $0,62(0,32-1,22)$ & & $0,60(0,30-1,19)$ & \\
\hline
\end{tabular}

OR IC95\% = odds ratio - intervalo de 95\% de confiança; * OR ajustado por idade, escolaridade e sexo

Tabela IV - Modelo de regressão múltipla: variáveis associadas aos idosos (+ de 60 anos) praticantes de atividade física no lazer. São Paulo, Vigitel-2017, n = 693.

\begin{tabular}{lcc}
\hline Variáveis e categorias & OR (IC95\%) & p \\
\hline Escolaridade (anos) & & $<0,001$ \\
$0-8$ anos & 1,00 & \\
$9-11$ anos & $1,53(0,94-2,48)$ & $<0,001$ \\
$>12$ anos & $2,47(1,58-3,85)$ & \\
Estado de saúde & & 1,00 \\
$\quad$ Ruim/muito ruim/não sabe & $1,27(0,46-3,46)$ \\
Regular & $2,96(1,12-7,76)$ \\
Bom & $5,14(1,86-14,23)$ & \\
Muito bom & & \\
\hline
\end{tabular}

OR IC95\% = odds ratio - intervalo de 95\% de confiança 
No entanto, em relação às características socioeconômicas, importante variável em estudos epidemiológicos ${ }^{(34)}$, a atual pesquisa apontou que idosos com escolaridade superior estão positivamente associados à AFLS, corroborando estudos envolvendo tanto população com idade igual e acima de 18 anos ${ }^{(18)} \operatorname{como}$ com idade $\geq 60$ anos ${ }^{(16,22,33)}$.

A adoção de um estilo de vida ativo no lazer reduz o risco de doenças crônico-degenerativas e, ainda, sua regularidade constitui um importante indicador de incorporação de hábitos de vida mais saudáveis ${ }^{(31,32)}$. Além de controlar e/ou evitar tanto os sintomas de doenças como a manutenção da aptidão física, a prática regular oferece, também, benefícios psicológicos, influenciando a autoavaliação da saúde ${ }^{(28)}$. A associação entre referir estado de saúde "muito bom" e "bom" e a atividade física de lazer aqui encontrada corrobora pesquisas nacionais ${ }^{(28)}$.

O presente estudo encontrou que quanto maior os anos de estudo, maior a probabilidade da redução das iniquidades em saúde, em especial nos grupos mais vulneráveis, dada a maior concentração das DCNT e seus fatores de risco na população de baixa renda e escolaridade. Chama a atenção a não associação da AFLS com participantes que referem algum tipo de DCNT e consumo de álcool, enquanto tais características são encontradas em outros estudos nacionais ${ }^{(22)}$. Destaca-se uma pesquisa envolvendo 1.950 idosos residentes em quatro áreas da região de São Paulo(2), que, mesmo após o ajuste por idade, observou maior prevalência de indivíduos idosos ativos no lazer com até três doenças crônicas quando comparados aos que possuem acima de quatro, e entre os que ingerem bebida alcoólica numa frequência de 1-3 vezes por semana.

Algumas limitações devem ser observadas quanto aos resultados do atual estudo. Aponta-se para o fato de o inquérito não trazer informações mais detalhadas sobre outros fatores que podem influenciar na prática de atividade física no lazer, como os fatores socioambientais e o acesso a espaços públicos adequados e seguros próximos a residência dos entrevistados. Além disso, observou-se que os parâmetros adotados de classificação na AFLS no presente estudo são padronizados para todos os grupos etários. Apesar dessas limitações, o presente estudo apresenta como ponto forte a inclusão de uma grande quantidade de indivíduos idosos residentes no município de São Paulo.

Os achados desta pesquisa contribuem para o monitoramento do comportamento saudável entre os idosos. No entanto se evidencia diferença na AFLS segundo o perfil sociodemográfico e o comportamento de saúde. Essas características precisam ser consideradas no planejamento e monitoramento das políticas de saúde e nas ações de incentivo à prática de atividade física. É mister salientar a necessidade de adequações às políticas de promoção e monitoramento da AFLS que expressem o conceito de desenvolvimento humano, considerando os fatores associados à longevidade humana.

\section{CONCLUSÃO}

No contexto geral, pode-se inferir que um a cada quatro idosos moradores do município de São Paulo praticam atividade física suficiente no lazer. Entre os idosos, a prevalência foi maior entre os mais jovens, do sexo masculino, com companheiro, branco, maior escolaridade, com ocupação, que relataram estado de saúde "muito bom", referiram não possuir doenças crônicas, consumir bebida alcoólica e ser não fumante.

As características com maior poder explicativo para atividade física suficiente no lazer foram a maior escolaridade e referir "muito bom" e "bom" estado de saúde. Tal fato reforça a necessidade de planejamento e monitoramento das políticas de saúde considerando as diferenças sociodemográficas e os comportamentos de saúde para a prática e/ ou manutenção da atividade física de lazer entre os idosos.

\section{AGRADECIMENTOS E CONFLITOS DE INTERESSE}

Os autores agradecem ao sistema de Vigilância de Fatores de Risco e Proteção para Doenças Crônicas por Inquérito Telefônico (Vigitel) pelo delineamento, coleta e disponibilidade dos dados.

Os autores declaram não haver conflitos de interesses.

\section{CONTRIBUIÇÕES}

Bruno Holanda Ferreira e Olinda do Carmo Luiz contribuíram com a elaboração e delineamento do estudo; a aquisição, análise e interpretação dos dados e a redação e/ou revisão do manuscrito. Bruna Gabriela Marques contribuiu com a aquisição, análise e interpretação dos dados e a redação e/ou revisão do manuscrito. 


\section{REFERÊNCIAS}

1. United Nations. World population prospects: key findings and advance tables. New York: United Nations; 2017.

2. Vasconcelos AMN, Gomes MMF. Transição demográfica: a experiência brasileira. Epidemiol Serv Saúde. 2012;21(4):539-48.

3. Phillip JM, Aifuwa I, Walston J, Wirtz D. The Mechanobiology of Aging. Annu Rev Biomed Eng. 2015;17(1):113-41.

4. Copeland JL, Ashe MC, Biddle SJ, Brown WJ, Buman MP, Chastin S, et al. Sedentary time in older adults: a critical review of measurement, associations with health, and interventions. $\mathrm{Br} J$ Sports Med. 2017;51(21):1-8.

5. World Health Organization. Strategy on diet, physical activity and health [Internet]. Geneva: WHO; 2004 [acesso em 2019 Set 15]. Disponível em: https://www.who.int/dietphysicalactivity/strategy/eb11344/strategy_ english_web.pdf

6. The Lancet. GBD 2017: a fragile world. Lancet. 2018;392(10159):1683.

7. Orkaby AR, Forman DE. Physical activity and CVD in older adults: an expert's perspective. Expert Rev Cardiovasc Ther. 2018;16(1):1-10.

8. Reiner M, Niermann C, Jekauc D, Woll A. Long-term health benefits of physical activity - a systematic review of longitudinal studies. BMC Public Health. 2013;13(813):1-9.

9. Levin $\mathrm{O}$, Netz Y, Ziv G. The beneficial effects of different types of exercise interventions on motor and cognitive functions in older age: a systematic review. Eur Rev Aging Phys Act. 2017;14(20):1-23.

10. Oliveira MA, Fernandes RS, Daher SS. Impacto do exercício na dor crônica. Rev Bras Med Esporte. 2014;20(3):200-3.

11. Minghelli B, Tomé B, Nunes C, Neves A, Simões C. Comparação dos níveis de ansiedade e depressão entre idosos ativos e sedentários. Rev Psiquiatr Clín. 2013;40(2):71-6.

12. Sieverdes JC, Ray BM, Sui X, Lee DC, Hand GA, Baruth M, Blair SN. Association between Leisure Time Physical Activity and Depressive Symptoms in Men. Med Sci Sport Exerc. 2012;44(2):260-5.

13. McPhee JS, French DP, Jackson D, Nazroo J, Pendleton N, Degens H. Physical activity in older age: perspectives for healthy ageing and frailty. Biogerontology. 2016;17(3):567-80.

14. Guthold R, Stevens GA, Riley LM, Bull FC. Worldwide trends in insufficient physical activity from 2001 to 2016: a pooled analysis of 358 population-based surveys with 1.9 million participants. Lancet Glob Health. 2018;10(6):e1077-86.

15. GBD 2017 Risk Factor Collaborators. Global, regional, and national comparative risk assessment of 84 behavioral, environmental and occupational, and metabolic risks or clusters of risks for 195 countries and territories, 1990-2017: a systematic analysis for the Global Burden of Disease Study 2017. Lancet. 2018;392(10159):1923-94.

16. Cruz MS, Bienal RTI, Claro RM. Tendência da prática de atividade física no lazer entre adultos no Brasil (2006-2016). Cad Saúde Pública. 2018;34(10):1-14.

17. Sousa NFS, Lima MG, Cesar CLG, Barros MBA. Envelhecimento ativo: prevalência e diferenças de gênero e idade em estudo de base populacional. Cad Saúde Pública. 2018;34(11):1-14.

18. Casas RCRL, Bernal RTI, Jorge AO, Melo EM, Malta DC. Fatores associados à prática de atividade física na população brasileira - Vigitel 2013. Saúde Debate. 2018;42(4):134-44.

19. Barbosa AR, Lebrão ML, Marucci MFN. Prevalência de inatividade física em idosos do Município de São Paulo. Lect Educ Fis Deportes. 2007;11(105):1-9.

20. Inácio RF, Salvador EP, Florindo AA. Análise descritiva da prática de atividade física no lazer de idosos residentes em uma região de baixo nível socioeconômico da zona leste de São Paulo, SP. Rev Bras Ativ Fís Saúde. 2011;16(2):150-55. 
21. Borim FSA, Barros MBA, Neri AL. Autoavaliação da saúde em idosos: pesquisa de base populacional no Município de Campinas, São Paulo, Brasil. Cad Saúde Pública. 2012;28(4):769-80.

22. Zaitune MPA, Barros MBA, César CLG, Carandina L, Goldbaum M, Alves MCGP. Fatores associados à prática de atividade física global e de lazer em idosos: inquérito de Saúde no Estado de São Paulo (ISA-SP), Brasil. Cad Saúde Publica. 2010;26(8):1606-18.

23. Ministério da Saúde (BR), Secretaria de Vigilância em Saúde, Departamento de Vigilância de Doenças e Agravos não Transmissíveis e Promoção da Saúde. Vigilância de fatores de risco e proteção para doenças crônicas por inquérito telefônico: estimativas sobre frequência e distribuição sociodemográfica de fatores de risco e proteção para doenças crônicas nas capitais dos 26 estados brasileiros e no Distrito Federal em 2017 , Vigitel 2017. Brasília: Ministério da Saúde; 2018.

24. Instituto Brasileiro de Geografia e Estatística. Censo demográfico 2010: Características da população e dos domicílios: resultados do universo [Internet]. Rio de Janeiro: IBGE; 2011 [acesso em 2019 Set. 17]. Disponivel em: https://www.who.int/dietphysicalactivity/strategy/eb11344/strategy_english_web.pdf

25. Fundação Sistema Estadual de Análise de Dados. Apresentação [Internet]. [data desconhecida] [acesso em 2020 Abr. 19]. Disponível em: http://produtos.seade.gov.br/produtos/projpop/index.php

26. Knuth AG, Hallal PC. Temporal trends in physical activity : a systematic review. 2009;6(5):548-59.

27. Mielke GI, Hallal PC, Malta DC, Lee IM. Time trends of physical activity and television viewing time in Brazil : 2006-2012. Int J Behav Nutr Phys Act. 2014;11(101):1-9.

28. Benedetti TRB, Borges LJ, Petroski EL, Gonçalves LHT. Atividade física e estado de saúde mental de idosos. Rev Saúde Pública. 2008;42(2):302-7.

29. Mourão ARC, Novais FV, Andreoni S, Ramos LR. Atividade física de idosos relacionada ao transporte e lazer, Maceió, Brasil. Rev Saúde Pública. 2013;47(6):1112-22.

30. Varo JJ, Martínez-González MA, De Irala-Estévez J, Kearney J, Gibney M, Martinez JA. Distribution and determinants of sedentary lifestyles in the European Union. Int J Epidemiol. 2003;32(1):138-46.

31. Sun F, Norman IJ, While AE. Physical activity in older people: A systematic review. BMC Public Health. 2013;13(1):1-17.

32. Andersen K, Mariosa D, Adami HO, Held C, Ingelsson E, Lagerros YT, et al. Dose - response relationship of total and leisure time physical activity to risk of heart failure: a prospective cohort study. Circ Hear Fail. 2014;7(5):701-8.

33. Oliveira DV, Lima MCC, Contessoto LC, Cremonez JC, Antunes MD, Nascimento JRA Jr. Fatores associados ao nível de atividade física de idosos usuários das academias da terceira idade. Acta Fisiatr. 2017;24(1):17-21.

34. Liberatos P, Link BG, Kelsey JL. The measurement of social class in epidemiology. Epidemiol Rev. 1988;10(1):87-121.

\author{
Endereço para correspondência: \\ Bruno Holanda Ferreira \\ Faculdade de Medicina da Universidade de São Paulo \\ Av. Doutor Arnaldo, 455 \\ Bairro: Cerqueira César \\ CEP: 01246-903 - São Paulo - SP - Brasil \\ E-mail: brunohf84@usp.br
}

Como citar: Ferreira BH, Marques BG, Luiz OC. Fatores associados à atividade física de lazer entre idosos do município de São Paulo. Rev Bras Promoç Saúde. 2020;33:10338. 